\title{
The Augmented Patient Chart: Seamless Integration of Physical and Digital Artifacts for Hospital Work
}

\author{
Myrna S. Zamarripa ${ }^{1}$, Victor M. Gonzalez ${ }^{2}$, and Jesus Favela ${ }^{1}$ \\ ${ }^{1}$ Department of Computer Science, CICESE Research Center, Mexico \\ ${ }^{2}$ School of Informatics, University of Manchester, United Kingdom \\ mzamarri@cicese.mx, vmgonz@manchester.ac.uk, favela@cicese.mx
}

\begin{abstract}
Even with the introduction of computer technology, paper-based artifacts remain ubiquitous in hospital settings. The need to manually transfer and update information from the physical to the digital realm is a common practice among hospital staff, which, although usually well managed, at times becomes a source for errors and inconsistencies. This paper presents an augmented patient chart system that preserves the use of paper and allows capturing information directly to the system through the use of a digital pen. An evaluation of the system with 22 volunteers indicates a significant reduction in the number of errors while reading information, a significant increment on the accuracy while annotating data, as well as a trend towards less time spent while annotating data on the digital paper. Based on our results, we argue that the design preserves the advantages associated with paper while increasing the availability of information and its trustworthiness.
\end{abstract}

Keywords: Ubiquitous computing, Hospital work, Physical artifacts, Digital paper.

\section{Introduction}

Even with the introduction of computer technology, paper-based artifacts remain ubiquitous in hospital settings. One of the reasons for paper being so prevalent is the high level of mobility experienced by hospital workers, whom have to move from place to place, carrying information on portable artifacts such as notebooks, clipboards, and information sheets [1]. In clear contrast to a desktop-based working environment [2] or central control rooms [5], the information in hospitals is not generally concentrated in a single place but it is distributed in different sites. The storage of information in physical paper artifacts, results on clear inefficiencies, as information cannot be retrieved promptly whenever and by whoever might need it. Furthermore, the need to manually transfer and update information from the physical to the digital realm is becoming a daily practice among hospital staff, which, although usually well managed, at times becomes a source for errors and inconsistencies. Estimates indicate that up to 98,000 people die in the US as a result of medical errors [4], which serves to realize the importance of proper information management in hospital settings. 
The use of portable computers and personal digital assistants (PDAs) could help support information management in such a mobile working environment, yet, it is clear that much is lost when paper affordances are left out. For instance, a study comparing the use of paper to PDAs or TabletPCs for the elaboration of medical notes showed that writing is faster when using pen and paper [7]. Consequently, rather than replacing paper, our aim is to use pervasive computing technology to seamlessly integrate paper into physical-digital hospital information systems. This integration of physical artifacts and digital information is one of the main challenges facing the field of ubiquitous computing.

We developed an augmented patient chart system, based on Anoto ${ }^{\mathrm{TM}}$ technology that preserves the use of paper and allows capturing information directly to the digital system through the use of a digital pen. The solution maintains the essence of the original patient chart and introduces features that make it richer by providing up to date information and more powerful graphical presentations. The system was designed to facilitate its integration to the information systems operating in a typical hospital (e.g. Electronic Patient Record, Clinical Decision Support Systems, Computerized Physician Order Entry Systems, etc).

The rest of the paper is organized in the following way: Section 1 presents some observations and requirements derived from a case study conducted to inform the design of the application. Section 3 discusses the application, its architecture and functionality. Section 4 presents results from an evaluation of the system by 22 nurse students. Finally, Section 5 closes the paper with our conclusions and discussion of future work.

\section{Hospital Patient Charts: A Case Study}

Our design emerged from an ethnographic study consisting of interviews and shadowing of people at a mid-size public Hospital in Ensenada, Mexico. We focused our study on operative nurses as they interact constantly with the patient chart. Using the patient chart, the operative nurses are in charge of following up and carrying out the indications left by physicians treating the patients. During three days, two nurses were followed (shadowed) during their entire shift to understand the relevance of the patient chart on executing their daily activities.

As a result of the observations we were able to identify:

- The work processes around the patient chart

- The general structure of the patient chart as well as the types of information it usually contains.

- The main users of the patient chart.

- The typical annotations and codes used by hospital workers on the patient chart.

- The physical location and level of mobility of the patient chart across the turn shift.

- Role of the patient chart on the activities carried out by operative nurses

- Other information artifacts used together with the patient chart. 
At the end of the shadowing, semi-structured interviews complemented the observations and allowed us to inquiry informants with regards to the limits of the current version of the patient chart and implications of potential changes to it. During the study we also collected many samples of patient charts that served to conduct further analysis after the observations.

\section{The Augmented Patient Chart}

The analysis of the previous version of the patient chart resulted on a design that preserves the essential elements of that version, eliminates some of its problems, and provides new ways to capture data by taking advantage of the digital pen. The augmented patient chart system was developed and organized into two main components. The first component is the actual paper format that serves to implement the version of the patient chart using the digital pen and Anoto ${ }^{\mathrm{TM}}$ technology. The second component is the software system that processes the information captured with the digital pen. This section describes the characteristics of the system and discusses some of the design decisions made to implement it.

\subsection{General Working Characteristics of the Digital Pen and Paper}

Based on the Anoto $^{\mathrm{TM}}$ technology, the system works with a digital pen with an embedded camera on it that takes pictures as the device moves across the surface while making annotations on the paper. The digital pen is just slightly bigger than a regular pen which means that people can use it without noticing its special characteristics. The paper contains a special printed dotted pattern that works with the camera to identify the exact position where the annotation is made on the paper. The area covered by the pattern used by Anoto ${ }^{\mathrm{TM}}$ allows creating a unique pattern for each physical form which allows designing applications consisting of multiple formats. For our particular case, we used a pattern for a letter-sized format (8.5 x 11 in).

\subsection{System's Mode of Interaction and Operation}

Our design of the augmented patient chart aimed at emphasizing the interaction with the paper format in order to preserve the natural work flow followed by nurses. Although some functions of the system (e.g. transferring data, or validating some entries) require the interaction with the computer-based interface, we defined the operation of the system in such a way that such interactions could be performed at the end of the working shifts. Figure 1 shows the new design proposed for the patient chart.

Following the original design, the frontal part of the augmented patient chart is organized into three main sections. At the top of the format a section is used to list patient's details such as name, age, gender, religion, and others. The middle section presents graphs for the cardiac pulse, internal and external temperature of the patient. Our design replaced manually generated graphs with a set of widgets to annotate accurate values used to plot graphs every time a new patient chart is created. The bottom section of the format is used to annotate (free format) additional information 
about the conditions observed. The rear part of the format includes a section to annotate prescribed medications, general observations and specifying messages to other co-workers attending the patient.

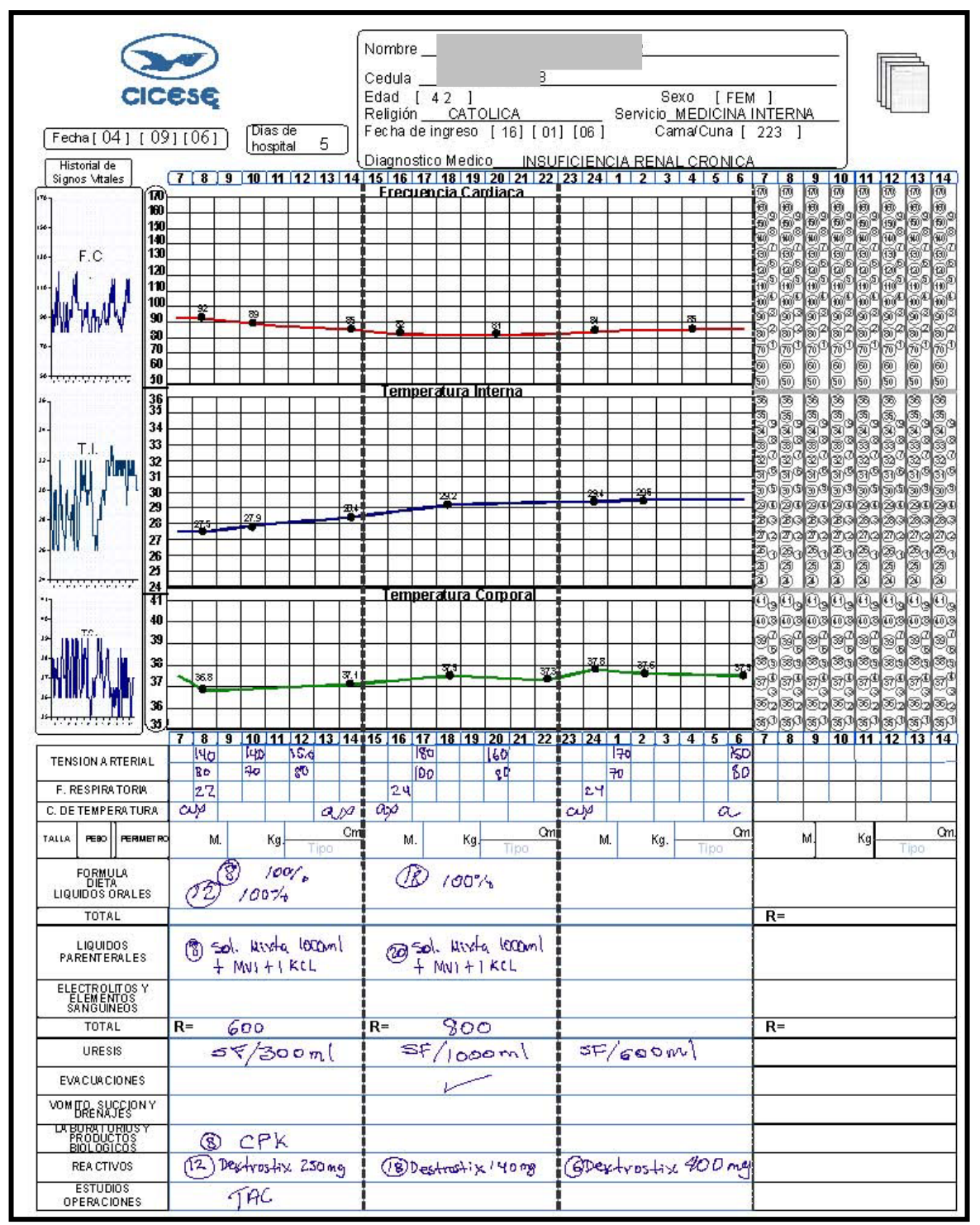

Fig. 1. The designed physical-digital patient chart (front)

The general operation of the system is detailed in the following lines: The nurse annotates information on the format as he does with the old version. Once he completes the annotations, he can leave the format and the digital pen and proceed 
with his work. We assume that in general, it will be at the end of the work shift when the digital pen is placed in its base and then the information captured on the format is transferred to the system. Clearly, in some other situations immediate transfer might be necessary (as when a message has to be sent or medication has to be ordered) and the digital pen will have to be placed in its base right away. Whatever the case, once the pen is placed in its base, all information in memory is transferred to the system.

When the application detects that a particular action was requested (e.g. printing a new format or sending a message), the action is sent to a module that executes it.

The PC to which the digital pen is attached hosts a module named PLS (Paper Lookup Service) that detects and directs the action to a particular application, based on the pattern of the paper. The application then explores the areas of the paper that have been modified, obtains the information and processes it accordingly. Based on the annotation, the application executes actions such as updating records in the database, sending a message (through an electronic mail client), requesting confirmation of prescription orders or printing a new patient chart (including processing the information required to generate new graphs). Figure 2 shows the sequence diagram showing hot the components of the system interoperate.

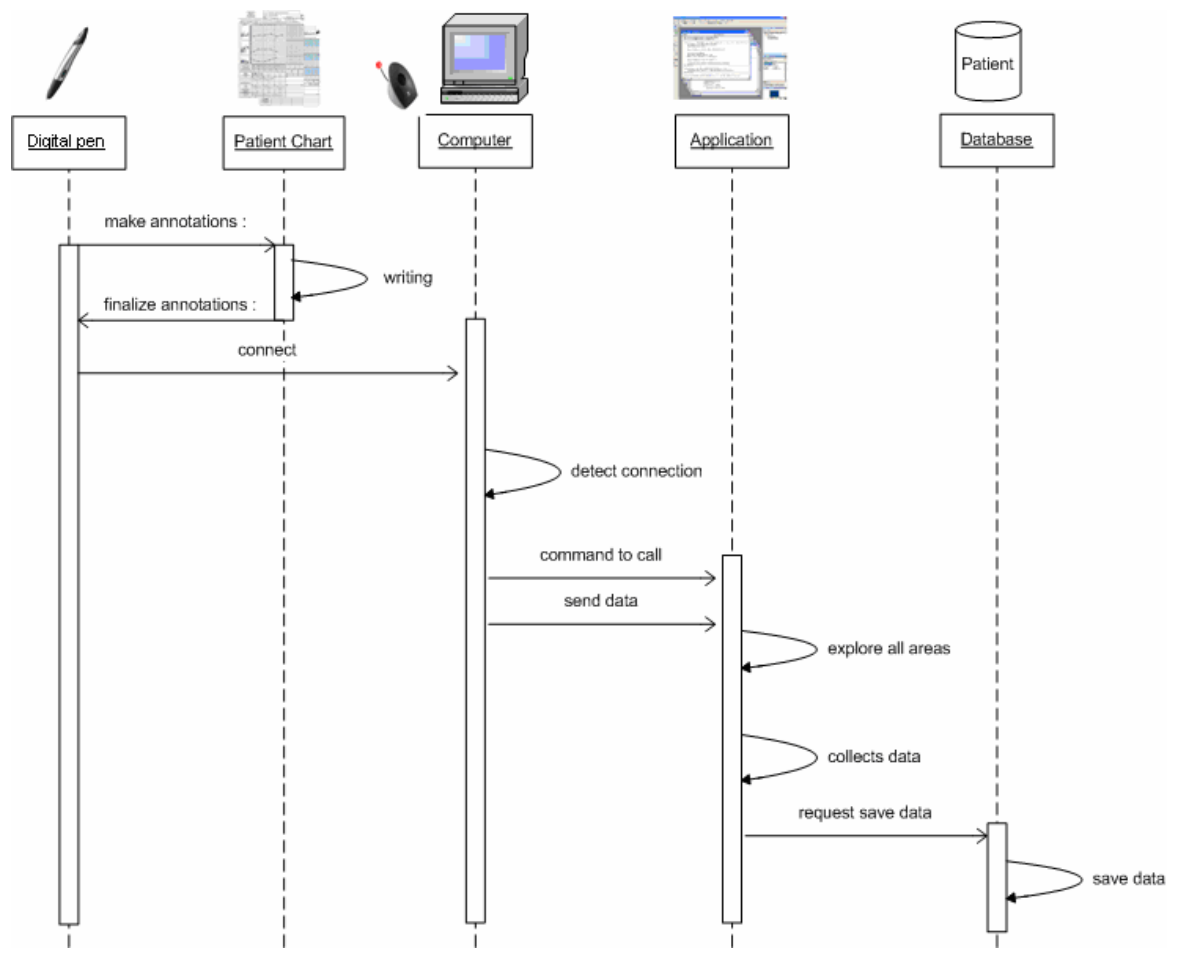

Fig. 2. Sequence diagram

In order to avoid errors, some annotations performed on the format have to be confirmed before an action takes place. Such is the case of the delicate task of ordering medication. Once the digital pen is placed in its base, the system detects 
whether the medication section was modified. If new medications were annotated the system uses the captured images of the annotations and presents them on the computer screen. The user then has to manually scroll down among the list of medications, as well as other details such as dosage and periodicity, to select the one matching the annotation (see Figure 3).

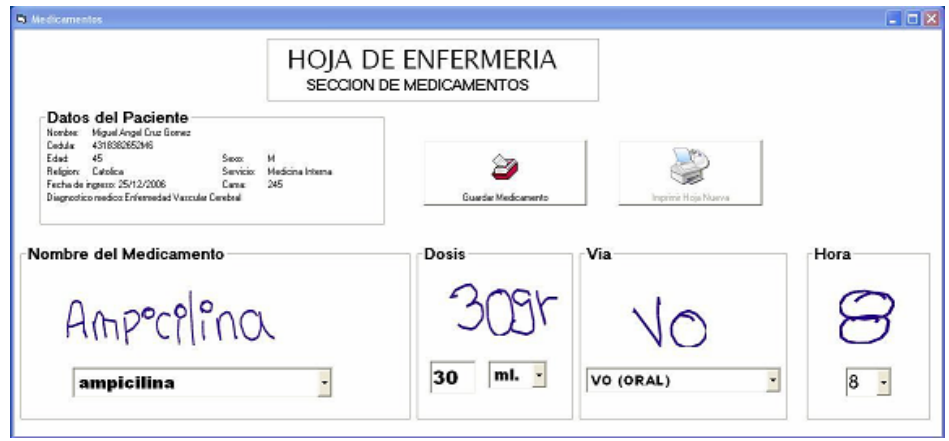

Fig. 3. Confirmation of prescriptions

\section{Evaluation}

As a result of preliminary tests with users, the design of the system experienced a number of modifications until it eventually reached a level of maturity that allowed us to conduct a formal evaluation which results are presented in this section.

\subsection{Purposes of the Evaluation and Methods}

We aimed at conducting the evaluation to assess the advantages of the augmented patient chart format against the previous format used by nurses. Thus the evaluation of the system was guided by three main hypotheses:

H1. The time required to consult information using the new format is less than when using the traditional format.

We considered that the characteristics of the new format would produce that result because the information is structured in a better way, some annotations from the previous working shifts is printed with standard font and the values of graphs are more precisely plotted.

H2. The writing time using the new format is similar than when using the traditional format.

Our rational for this hypothesis was that the digital pen will not prove to be more challenging to use than the traditional one. Therefore, the time to write annotations would be very similar with both formats.

H3. The number of errors while either writing or finding information in the format will be less with the new format than with the traditional one. 
We believed that this hypothesis would be true because the new format has a better structure, layout and leads to a better and more precise understanding of the information.

The three hypotheses above were tested in a controlled evaluation of the system. We decided to replicate as much as possible the conditions experienced by nurses while using the patient chart at the hospital. Thus, we designed our tasks and the evaluation setting with that purpose. The system was evaluated by 22 nurse students in Ensenada, Mexico (Figure 4). The nurse students were familiar with traditional patient charts because they have to spend practical hours at the local hospital as part of their education. After an initial training, the participants were asked to complete a set of representative tasks using both the traditional patient chart and the new version designed for the digital pen.

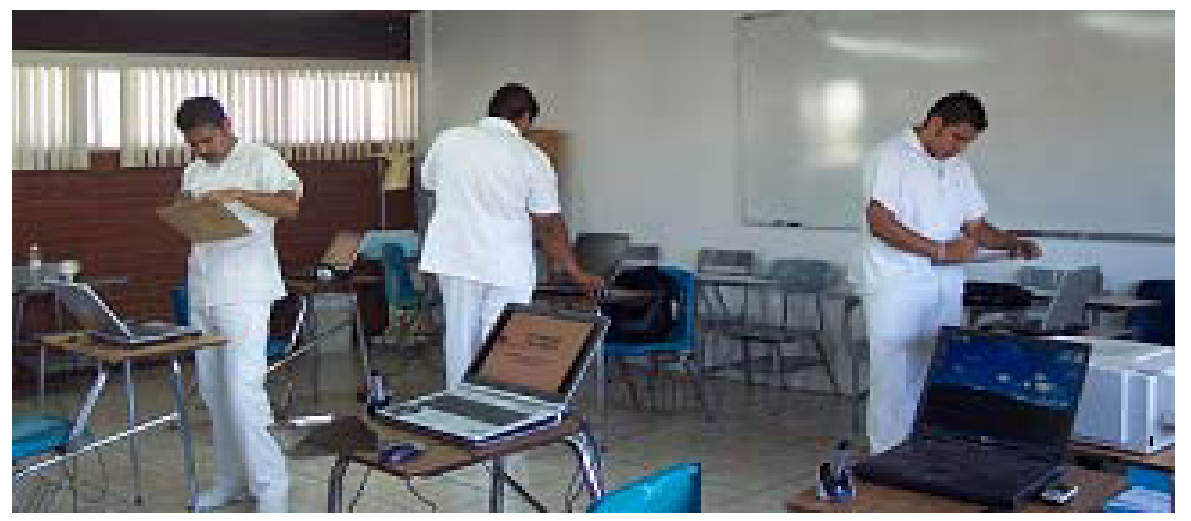

Fig. 4. Nurse students evaluating the system

The main working tasks that our evaluation aimed at exploring were: (1) reading of information on the format that was captured by another colleague during the previous work shift, and (2) annotation of information on the format as it would be done during the interaction with the patient; both tasks were performed by each participant using the traditional and the new patient chart formats. In order to minimize learning effects, we divided the participants and assigned them randomly to one of two groups. The first group (12 participants) started the evaluation using the digital format, and then used the traditional one. The second group (10 participants) started with the traditional format and then switched to the digital.

The evaluation was conducted in the following phases:

Phase 1. Using a laptop computer, each participant watched a video presentation describing the technology, as well as the set of tasks that they would be asked to complete. At the end of the presentation, the participant had the opportunity to clarify doubts or solve any question with the researcher.

Phase 2. During this phase the participants moved to another laptop computer and were given a copy of the augmented patient chart format. Standing in front of the computer, the participants have to respond a computer-based questionnaire (15 
questions) on the computer by using the information contained on the patient chart format. This was the consulting information task.

Phase 3. After completing the previous questions, the participants moved to another computer and stood in front of it. They were asked to read information appearing on the computer screen that they have to use to annotate some sections of the patient chart format ( 7 tasks). After this, the nurses were asked to place the digital pen inside its base and then proceed to complete a confirmation of some of the information they gathered. This was the writing information task.

Phase 4. At this point, the participants received a copy of the traditional patient chart and moved to another computer to complete similar tasks as if they were dealing with another patient. Using the format they were asked to respond 14 questions appearing on the computer screen. It is important to clarify that the questions they have to respond during these phase were different to the ones they responded in a phase 2 since they were related to a different patient.

Phase 5. During this phase the participants move to the last computer station in the room and following the instruction on the computer they were asked to fill information on the format ( 7 tasks).

Phase 6. At the end of the evaluation, participants completed questionnaires based on TAM (Technology Adoption Model) [3] that served to explore their perspectives with regards to the adoption of our solution.

The first 12 participants followed the order described above. The next 10 participants completed the task in the following sequence: phase 1, phase 4, phase 5, phase 2, phase 3 and phase 6 .

\section{Results}

The results of the evaluation indicate that using the new patient chart format participants experienced a significant reduction in the number of errors while reading information related to vital signs. Nine questions were asked to be responded by reading the graphs with vital sign information of the patient. On average, those volunteers using the traditional patient chart obtained significantly less correct answers (6.05 s.d. 1.35) compared to those using the new patient chart format (7.5, s.d. 1.79) $(\mathrm{t}=3.864, \mathrm{p}<0.001)$. The other two sections of the format (evaluation and prescription of medicines) produced no significant differences in the number of errors when using either format.

We also found a significant increment of the accuracy while responding to the three tasks involving the annotating of data related to vital signs. On average the volunteers using the new patient chart format had more correct answers $(5.18$, s.d. 1.68) than those using the previous version of the format $(3.68$, s.d. 2.70) $(t=2.28$, $\mathrm{p}<0.05)$. No advantage was observed for either version of the format while responding to tasks related to patient evaluation or prescription of medicines.

A trend towards less time spent while annotating data on the digital paper was observed. On average, those participants using the previous version of the format spend slightly more time $(9.74 \mathrm{~min}$. s.d. $3.01 \mathrm{~min}$.) completing the tasks than those using the new digital format (9.10 min s.d. $2.52 \mathrm{~min})$. Clearly, the difference is small, but still provides some evidence of the advantages of the new design. 
Furthermore, based on the results from the TAM questionnaire, we found that the perceptions of participants with regards to the augmented patient chart's usefulness are very encouraging: they recognize the advantages of the solution, but more important, the new interface does not appear to be either challenging or fundamentally modifying the essence of the task performed with the artifact.

\section{Conclusions}

The use of physical paper artifacts working in combination with automated digital systems has been reported to be common not just in traditional office work contexts [6], but also as part of the practices of hospital and medical workers [2].

We proposed, implemented, and evaluated an augmented patient chart supporting a seamless integration of physical and digital artifacts for hospital work. Among its characteristics, the solution replaces manually generated graphs of patient's vital signs (cardiac pulse, internal and external temperature) with a set of widgets to annotate accurate values used to plot graphs every time a new patient chart is created. The system allows the free annotation of data such as medications that are validated when these are transferred to the hospital information systems. It also provides ways to annotate messages to co-workers and transfer them electronically. By combining widgets with pre-defined options and free annotations the system optimizes data capture without the need of explicit character recognition.

An evaluation of the patient chart system with 22 participants indicates a reduction in the number of errors while reading information, a significant increment on the accuracy while annotating data, as well as a trend towards less time spent while annotating data on the digital paper. The results of the TAM questionnaires indicate that users are inclined to adopt the system. Comments made by participants during and after the evaluation served to understand that users felt comfortable with the proposed design of the patient chart because it looks very similar to the original version and preserves the use of paper and pen as the main way to handle the information. These results allow us to argue that our design would be able to preserve the advantages associated with paper while increasing the availability of information and its trustworthiness.

Based on our understanding of hospital needs, we can identify a set of benefits resulting from using the type of technology we are proposing. Firstly, the level of precision and clarity is clearly increased which can, in principle, contribute towards the reduction of errors while managing information. Secondly, as a result of the architecture of the system and the way it manages the data, the digital format can be integrated and operate with other hospital information systems (e.g. Electronic Patient Record, Clinical Decision Support Systems, Computerized Physician Order Entry Systems, etc) without major modifications. Thirdly, because data is preserved in digital and physical versions it allows recreating the formats (and hand written annotations) in case of damage or lost. Finally, because the information is stored in databases it can be consulted without retrieving the physical artifact.

As part of our future work we plan to extend the functionality of the system and conduct an in situ extended trial of the system at a local hospital. 
Acknowledgments. We wish to express our gratitude to all the volunteer nurse students that took part during the different stages of the design of the augmented patient chart, as well as to Raul Romero for his support organizing the evaluation, and to Monica Tentori, Elisa Moran, Leonardo Galicia, Daira Sanchez and Mario García for their support during the evaluation of the system.

\section{References}

1. Bardram, J., Bossen, C.: Mobility Work: The Spatial Dimension of Collaboration at a Hospital. Journal of Computer Supported Cooperative Work (Springer, Netherlands) 14(2), 131-160 (2005)

2. Bossen, C.: The Parameters of Common Information Spaces: the Heterogenity of Cooperative Work at a Hospital Ward. In: Proceedings of ACM Conf. on Computer Supported Cooperative Work, CSCW, pp. 176-185. ACM Press, New York (2002)

3. Davis, F.: Perceived usefulness, perceived ease of use, and user acceptance of information technology. MIS Quarterly 13(3), 319-339 (1989)

4. Kohn, L., Corrigan, J., Donaldson, M. (eds.): To Err is Human: Building a Safer Health System. Committee on Quality of Health Care in America. Institute of Medicine. Washington, DC, USA (1999)

5. Theureau, J., Filippi, G.: Analysing cooperative work in an urban traffic control room for the design of a coordination support system. In: Workplace Studies: Recovering Work Practice and Informing System Design, pp. 68-91. Cambridge Press, Cambridge (2000)

6. Sellen, A.J., Harper Richard, R.: The Myth of the Paperless Office. 56 MIT Press, Cambridge, Mass (2000)

7. Silva, J., Zamarripa, M., Strayer, P., Favela, J., Gonzalez, V.: Empirical Evaluation of a Mobile Application for Assisting Physicians in Creating Medical Notes. In: the Proceedings of the 12th Americas Conference on Information Systems. Acapulco, Guerrero, Mexico (2006) 